\title{
Transfert de fréquence par fibre optique en métrologie
}

\section{Frequency transfer using optical fiber in metrology}

\author{
Giorgio SANTARELLI ${ }^{1}$, Olivier LOPEZ ${ }^{2}$, Fabien KÉFÉLIAN ${ }^{2}$, Haifeng JIANG ${ }^{1}$, Bruno CHANTEAU ${ }^{2}$, \\ Michel LOURS ${ }^{1}$, François NARBONNEAU ${ }^{1}$, Adil HABOUCHA ${ }^{1}$, Scott CRANE ${ }^{1}$, Christophe DAUSSY ${ }^{2}$, \\ Anne AMY-KLEIN ${ }^{2}$ et Christian CHARDONNET ${ }^{2}$
}

\footnotetext{
${ }^{1}$ LNE-SYRTE, Observatoire de Paris, CNRS, UPMC, 61 Avenue de l'Observatoire, 75014 Paris, France, info.lne-syrte@obspm.fr. ${ }^{2}$ Laboratoire de Physique des Lasers, Université Paris 13, Sorbonne Paris Cité, CNRS, 99 Avenue Jean-Baptiste Clément, 93430 Villetaneuse, France.
}

\begin{abstract}
Résumé
L'utilisation de la fibre optique pour la comparaison et la distribution de références ultrastables de fréquence s'est fortement développée ces dix dernières années. Le LNE-SYRTE et le LPL sont des laboratoires pionniers dans l'étude et le développement de ces techniques. La stabilité de fréquence et l'exactitude des liens optiques fibrés surpassent désormais les performances des méthodes classiques qui exploitent les satellites GPS ou géostationnaires. Dans cet article nous présentons une revue des méthodes et des résultats obtenus. Nous montrons également qu'il est possible d'utiliser les réseaux fibrés de l'internet pour réaliser la comparaison et la distribution des signaux métrologiques ultrastables sur de longues distances. La stabilité et l'exactitude de tels liens sur réseaux fibrés de l'internet sont aujourd'hui proches des limites que l'on peut attendre sur une fibre optique dédiée à cette application. Cette nouvelle technique ouvre la voie au déploiement au niveau national et international d'un réseau optique métrologique ultrastable.
\end{abstract}

MOTS CLÉS : TEMPS, FRÉQUENCE, LIEN OPTIQUE FIBRÉ, LASER STABLE.

\footnotetext{
Abstract

The distribution and the comparison of an ultrastable reference frequency using optical fibres have been greatly improved in the last ten years. LNE-SYRTE and LPL (University Paris 13) have been pioneering the development of these new methods. In this paper we present a review of the methods and the results obtained. The frequency stability and accuracy of optical links surpass the well-established methods using GNSS and geostationary satellites. In this paper we show that it is possible to use public telecommunication network carrying Internet data to compare and distribute ultrastable metrological signals over long distances. In addition both frequency stability and accuracy are equivalent to those obtained with links developed with a dedicated fibre
}

network. This novel technique paves the way to the deployment of a national and continental wide ultrastable metrological optical network.

KEYWORDS: TIME, FREQUENCY, OPTICAL LINK, STABLE LASER.

\section{Introduction}

La métrologie des fréquences s'est rapidement développée depuis quinze ans en profitant notamment des avancées scientifiques majeures dans les domaines du refroidissement d'atomes par laser (prix Nobel de physique en 1997), et dans celui des méthodes de comparaison des étalons de fréquence, grâce à l'utilisation des lasers femtosecondes comme «peigne » de fréquence (prix Nobel de physique en 2005). Les meilleurs étalons de fréquences micro-ondes, qui reposent sur des fontaines atomiques, atteignent désormais une exactitude de quelques $10^{-16}$ en valeur relative. Les étalons de fréquence fondés sur des transitions atomiques dans le domaine optique visent des exactitudes relatives de $10^{-17}$ ou mieux encore [1-7].

Les performances exceptionnelles d'exactitude et de stabilité des étalons de fréquence modernes en font des outils particulièrement adaptés pour tester les théories de la relativité restreinte et générale à des niveaux inégalés ou plus largement pour réaliser des tests de physique fondamentale. C'est ainsi que la comparaison de différents types d'horloges dont les transitions atomiques ou 
moléculaires font intervenir différentes constantes fondamentales permet de mesurer d'éventuelles variations au cours du temps de ces dernières (par exemple le rapport des masses de l'électron et du proton). Ces expériences de laboratoire sont actuellement parmi les seules à pouvoir mettre à l'épreuve les théories développées au-delà du modèle standard [8-11].

Les comparaisons d'étalons de fréquence et/ou d'horloges se heurtent à la difficulté de transporter l'information sans dégradation lorsque les horloges sont distantes de centaines voire de milliers de kilomètres. Actuellement ces comparaisons sont effectuées via un ou des satellites en utilisant le système Global Positioning system (GPS) ou des satellites de télécommunications. Malgré leur haut niveau de maturité, les performances de telles comparaisons sont actuellement limitées à un niveau proche de $10^{-15}$ en valeur relative pour un jour de mesure [12]. Cette résolution est insuffisante pour comparer les performances ultimes des meilleurs étalons atomiques microondes ou les nouveaux étalons de fréquence optiques. La possibilité d'améliorer de telles comparaisons par satellite est tributaire de la disponibilité d'un équipement en orbite dédié à cette application et est donc extrêmement coûteuse.

Depuis quelques années, la distribution de fréquences des étalons de fréquence par fibres optiques a connu des développements considérables et pourrait devenir une réponse à ce verrou technologique. Le cahier des charges est très clair : il s'agit de distribuer la fréquence de référence avec une résolution meilleure que $10^{-17}$ sur une journée de mesure entre laboratoires distants (typiquement de $1000 \mathrm{~km}$ ), soit un ordre de grandeur de mieux que la reproductibilité des meilleures horloges optiques du moment.

\section{Principe du transfert de fréquence par fibre optique}

Dans les années 2000, le LNE-SYRTE et le Laboratoire de Physique des Lasers (LPL) ont établi une collaboration afin de développer des liens optiques pour la comparaison d'étalons et la distribution de fréquences. Cette collaboration s'est d'abord appuyée sur une paire de fibres optiques de $43 \mathrm{~km}$ du réseau de télécommunications France Télécom reliant le LPL au LNE-SYRTE et entièrement dédiée à cette application. Un point essentiel de notre travail a consisté à corriger le bruit de phase apporté par la liaison optique, bruit dû aux variations de longueur optique de la fibre. Cette compensation active du bruit de transmission est réalisée grâce à la mesure du signal après un aller-retour dans la fibre. Dans les premières applications, une fréquence radio (RF) ou micro-onde - facilement raccordable à l'étalon primaire de fréquence - était transmise sous la forme d'une modulation d'amplitude de la porteuse optique [13,14]. Nous avons ainsi obtenu un transfert de fréquences RF et microondes avec une stabilité de $10^{-15}$ sur une durée de $1 \mathrm{~s}$ et de $10^{-18}$ sur une journée. Ces résultats nous ont alors placés en position de leader au niveau mondial pour le transfert RF à moyenne distance [15-18].
Nos résultats novateurs ont stimulé le développement de liens optiques dans les autres laboratoires de métrologie (essentiellement aux Etats-Unis, en Allemagne, en Angleterre, en Italie et au Japon) [19, 20]. La recherche s'est alors orientée vers la mise en œuvre de liens de longues distances, afin de démontrer une réelle alternative aux liens satellitaires. Avec le développement des peignes de fréquences et des horloges optiques, tous les acteurs de la recherche dans ce domaine ont convergé sur le principe d'une liaison purement optique, dans laquelle la référence de fréquence transmise est la porteuse optique elle-même. Le lien optique transporte ainsi une oscillation à $194 \mathrm{THz}$ issu d'un laser $(1,54 \mu \mathrm{m})$ dont la fréquence est stabilisée à un niveau métrologique, ce qui s'avère plus simple à mettre en œuvre et plus performant.

Dans ce cadre, une équipe franco-allemande ainsi qu'un groupe américain ont obtenu indépendamment les premiers résultats sur des liens purement optiques [21]. Nous avons ensuite démontré une liaison optique de $540 \mathrm{~km}$ utilisant le réseau Internet [22]. En Allemagne, le Physikalisch-Technische Bundesanstalt (laboratoire national de métrologie, à Braunschweig) et le Max-Planck-Institut für Quantenoptik (à Garching, près de Munich) viennent de réaliser une liaison optique d'une longueur record de $920 \mathrm{~km}$ en utilisant une liaison fibrée dédiée [23]. Ces avancées technologiques récentes ont ainsi démontré la faisabilité d'un réseau sur longue distance pour des applications métrologiques. Il existe donc maintenant une réelle opportunité pour le développement, à l'échelle européenne, d'un réseau entre les laboratoires de métrologie temps-fréquence (typiquement distants de $1000 \mathrm{~km}$ à $1500 \mathrm{~km}$ ).

Lors du transfert optique par fibre, le signal est dégradé par différentes sources de bruits thermiques et mécaniques. Les fluctuations de température de l'environnement ont une incidence principalement sur la vitesse et la longueur de propagation du signal dans la fibre, alors que les vibrations mécaniques affectent localement la fibre et se traduisent par une modification de la longueur physique de la fibre. Dans les deux cas, la conséquence est une variation du temps de propagation de l'onde optique et donc une fluctuation de la phase du signal transmis. Pour corriger ces fluctuations il est nécessaire de comparer le signal injecté avec celui ayant effectué un allerretour dans la fibre : la différence de la phase entre ces signaux contient le bruit de propagation aller-retour. La compensation des fluctuations de phase repose sur l'application d'une pré-correction au signal injecté dans la fibre, égale à la moitié de l'opposé de la différence de phase mesurée. Ceci est valable seulement si les parcours optiques à l'aller et au retour sont strictement équivalents (en termes de bruit ajouté par la liaison). Il est alors possible de compenser le bruit du support de transmission en temps réel. La bande passante de correction est limitée par le temps d'aller-retour du signal optique. Comme indiqué plus haut, cette méthode se décline en deux volets : le transfert par modulation d'amplitude radiofréquence/hyperfréquence ou par détection de la phase du champ électrique d'une porteuse optique. Nous allons expliciter ces deux approches dans les chapitres suivants. 


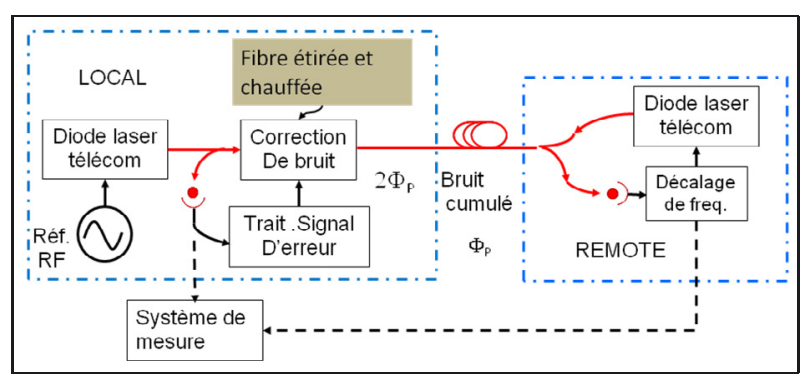

Fig. 1. - Schéma de principe du transfert de fréquence par modulation d'amplitude.

\section{Transfert radiofréquence et micro-onde}

Ce paragraphe est consacré à une brève description des méthodes et des résultats liés au transfert de fréquence par modulation d'amplitude d'une porteuse optique. Le principe de fonctionnement est présenté sur la figure 1. Le signal métrologique radiofréquence ou microonde à transmettre est superposé au courant de polarisation d'une diode laser à réseau de Bragg pour les télécommunications ou bien injecté dans un modulateur électro-optique d'amplitude de type Mach-Zehnder ou encore un modulateur à électro-absorption externe à la diode laser. Le signal optique ainsi modulé en intensité est envoyé dans la fibre optique et détecté, après propagation, par une photodiode sur le site distant. Le signal reçu, éventuellement décalé en fréquence, est alors utilisé pour moduler une seconde diode laser qui génère le signal retour injecté dans la fibre. Après ce parcours aller-retour il est possible d'obtenir un signal d'erreur et de corriger le bruit de transmission. Il est à noter que cette technique repose sur une détection quadratique du signal si bien qu'une atténuation de $10 \mathrm{~dB}$ du signal optique se traduit par une atténuation de $20 \mathrm{~dB}$ du signal radiofréquence.

Deux approches peuvent être envisagées pour la correction : l'une consiste à développer à partir du signal d'erreur un système de traitement électronique élaboré afin d'appliquer un déphasage au signal radiofréquence de départ exactement opposé à celui qui va affecter la phase du signal optique lors de la propagation aller dans la fibre, soit la moitié, au signe près, de l'erreur détectée. Une deuxième approche qu'on appellera optoélectronique, consiste à stabiliser directement le temps de propagation de l'onde transmise dans la fibre optique. Ceci rend le système moins complexe d'un point de vue électronique. Pour cela, une bobine de fibre optique est placée en série de la fibre de transmission. Cette bobine est chauffée (correction lente) et étirée (correction rapide) pour stabiliser la longueur optique de l'ensemble. Une simple détection de phase avec le signal de référence fournit le signal d'erreur qu'il s'agit d'annuler. Pour évaluer la performance du lien stabilisé ainsi réalisé il faut que les deux extrémités du lien, communément dénommées LOCAL et REMOTE, soient au même endroit. Un système de mesure complète le dispositif expérimental.

Avec ces deux techniques nous avons réalisé de nombreuses expériences de transfert de fréquence ultrastable sur la paire de fibres optiques dédiée de longueur

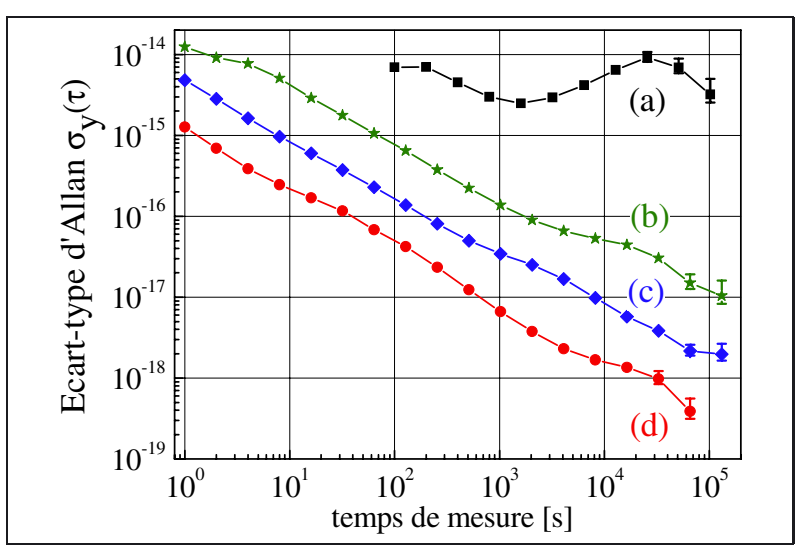

Fig. 2. - Stabilité de fréquence du lien optique LNE-SYRTE LPL pour différentes fréquences de modulation et longueurs de transmission, dans une bande de mesure de $5 \mathrm{~Hz}$ : (a) lien optique libre (carrés noirs), (b) $100 \mathrm{MHz}$ sur $43 \mathrm{~km}$ (étoiles vertes), (c) $1 \mathrm{GHz}$ sur $86 \mathrm{~km}$ (carrés bleus), (d) $9,15 \mathrm{GHz}$ sur $86 \mathrm{~km}$ (points rouges).

$2 \times 43 \mathrm{~km}$ reliant le LNE-SYRTE et le LPL. La toute première expérience a été réalisée à $100 \mathrm{MHz}$ sur une distance de $43 \mathrm{~km}[15,16]$. Ensuite nous avons augmenté la fréquence à $1 \mathrm{GHz}$ puis à $9,15 \mathrm{GHz}$ et réalisé un lien sur $86 \mathrm{~km}$ en plaçant les deux fibres en série puis sur $176 \mathrm{~km}$ en complétant par des bobines de fibres $[17,18]$. La figure 2 présente les résultats obtenus dans ces différentes configurations. La stabilité de fréquence est de 1 à $2 \times 10^{-14}$ à $1 \mathrm{~s}$ pour le lien à $100 \mathrm{MHz}$ et $1-2 \times 10^{-15}$ à $1 \mathrm{~s}$ pour le lien à $9,15 \mathrm{GHz}$. A $10000 \mathrm{~s}$, les stabilités sont respectivement de $8 \times 10^{-17}$ et de 1 à $2 \times 10^{-18}$. Les stabilités à la journée sont de l'ordre de $10^{-17}$ et quelques $10^{-19}$ pour les liens à $100 \mathrm{MHz}$ et $9,15 \mathrm{GHz}$ respectivement. Le lien à $1 \mathrm{GHz}$ montre des résultats intermédiaires. Les meilleurs résultats ont été obtenus avec le système optoélectronique en implémentant un brouillage de la polarisation. Ces méthodes sont désormais largement utilisées et ont récemment été perfectionnées par un groupe de recherche polonais qui a réalisé un lien optique de $420 \mathrm{~km}$ avec des diodes laser modulées en amplitude et des amplificateurs optiques bidirectionnels sur une liaison à très faibles pertes optiques [24].

\section{Transfert d'une fréquence optique}

Le transfert de fréquence par modulation d'amplitude est relativement simple à réaliser et les performances en termes de stabilité de fréquence peuvent permettre en principe la comparaison à distance des meilleurs étalons de fréquence micro-ondes. Cependant la construction d'un lien sur longue distance qui présente une forte atténuation optique pose problème. En effet la technique de modulation d'amplitude d'un faisceau laser ne paraît pas pouvoir être simplement étendue à des distances continentales en raison de l'atténuation encore plus forte du signal de modulation comme indiqué au paragraphe précédent. Une approche différente consiste à transmettre la phase du signal optique lui-même [25]. Une réduction considérable de la sensibilité à l'atténuation est alors obtenue par l'augmentation de près de cinq ordres de 


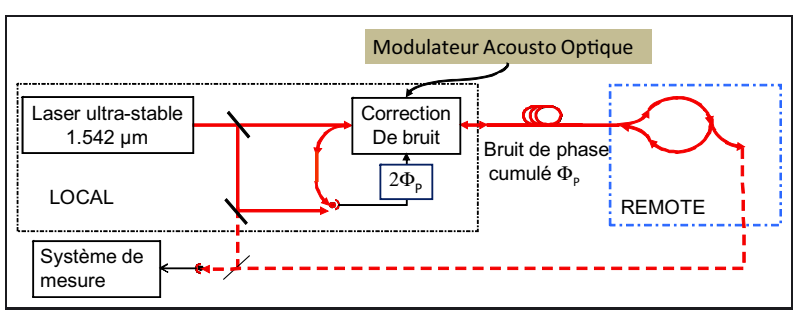

Fig. 3. - Schéma du principe de transmission sur fibre optique par détection de phase de la porteuse optique. Le MAO du laboratoire REMOTE n'est pas représenté par simplicité.

grandeur de la fréquence du signal utile et l'utilisation d'une détection hétérodyne. Au milieu des années 1990, un lien optique fibré de 3,5 km avait déjà été établi entre le BNM-LPTF (l'actuel LNE-SYRTE) et le laboratoire Kastler Brossel (LKB) à l'Université Paris 6. Cette liaison avait permis la transmission d'une référence optique à $780 \mathrm{~nm}$ pour la mesure de la fréquence d'une transition à deux photons du rubidium 87 [26].

La transmission de références optiques par fibre a considérablement gagné en intérêt depuis le développement d'étalons de fréquences optiques et de peignes de fréquence femtoseconde. Le principe de fonctionnement de ce type de lien optique est schématisé sur la figure 3. Un laser ultrastable, dont la largeur spectrale est inférieure au hertz, est connecté à la fibre optique à l'aide d'un circulateur ou d'un coupleur optique. Un modulateur acousto-optique (MAO) est utilisé pour décaler la fréquence optique et réaliser la correction du bruit de liaison. Après propagation dans la fibre, au niveau du site distant, une fraction du signal optique est prélevée par l'utilisateur et l'autre est réfléchie vers le site émetteur à l'aide d'un miroir ou d'un circulateur. Dans le site distant, un second MAO décale la fréquence optique, afin de distinguer le signal qui a effectué l'aller-retour des possibles réflexions parasites.

Dans le site de départ, le battement entre le signal optique ayant effectué la propagation aller-retour et le laser local est mesuré. Cette détection hétérodyne génère un signal radiofréquence à la fréquence double de la somme des fréquences appliquées aux deux MAO. Le signal détecté porte le bruit de phase équivalent au double des fluctuations du temps de propagation dans la fibre lors d'un aller simple. Ce signal permet de réaliser une boucle à verrouillage de phase qui corrige le bruit de phase du signal aller-retour en agissant sur le décalage en fréquence du MAO local. Cette boucle corrige du même coup le bruit de phase du signal aller dans le laboratoire distant car celui-ci reçoit bien une correction moitié de celle du signal aller-retour. Contrairement à l'asservissement effectué avec des bobines de fibres en transfert radiofréquence, l'asservissement par MAO stabilise la vitesse de phase du signal optique dans la fibre et non la vitesse de groupe. En s'appuyant sur cette technique nous avons réalisé en 2006 le premier lien tout optique sur une distance de $86 \mathrm{~km}$ sur la fibre reliant le LNE-SYRTE et le LPL puis sur $211 \mathrm{~km}$ en ajoutant $125 \mathrm{~km}$ de bobines de fibres. Cette expérience pionnière a été réalisée en collaboration avec le PTB [21]. Les résultats en termes

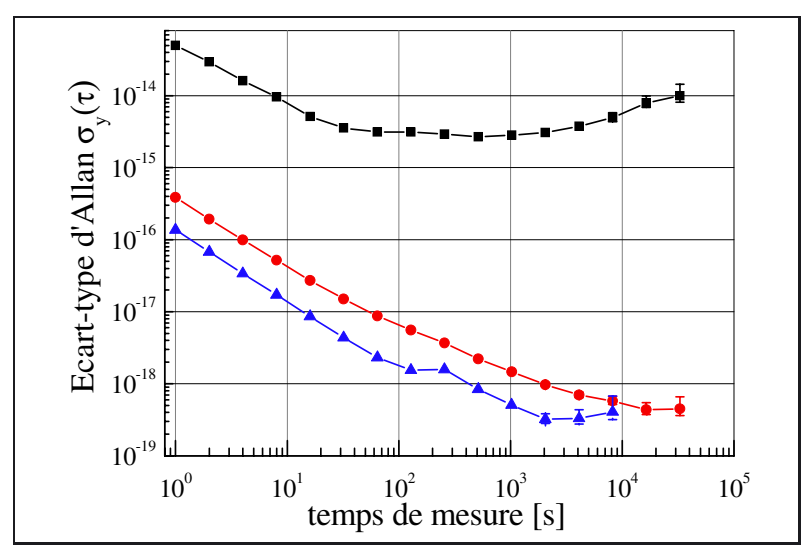

Fig. 4. - Stabilité de fréquence des liens optiques de 86 km et $172 \mathrm{~km}$. Lien non stabilisé (carrés noirs), lien stabilisé de $86 \mathrm{~km}$ (triangles bleus), lien stabilisé de $172 \mathrm{~km}$ (points rouges).

de stabilité de fréquence, $2 \times 10^{-15}$ sur 1 s et $6 \times 10^{-18}$ sur $10^{4} \mathrm{~s}$, sur $86 \mathrm{~km}$, ont démontré le fort potentiel de cette méthode. Peu après une équipe du NIST (National Institute for Standards and Technology) a réalisé un lien stabilisé de $251 \mathrm{~km}$ (75 km de fibre installée +176 km de bobines de fibres) avec des résultats remarquables et l'utilisation d'amplificateurs optiques bidirectionnels à fibre dopée erbium. Cette équipe a mis en évidence qu'au-delà de la réduction de la bande passante de correction, le délai lié à la distance entre le correcteur et le signal utile limite directement la capacité de l'asservissement à réduire le bruit du lien, pour des fréquences de Fourier beaucoup plus basses que la bande passante d'asservissement. L'amplitude maximale de réjection du bruit de propagation du lien est ainsi inversement proportionnelle à la longueur de la fibre, tandis que l'amplitude de bruit à corriger est elle typiquement proportionnelle à la racine carrée de la longueur [27]. Suite à notre expérience préliminaire nous avons perfectionné le dispositif en développant des lasers ultrastables à la longueur d'onde de 1542 nm (fenêtre de transmission optimale des télécommunications optiques) et en améliorant le système fibré de mesure de la phase optique. Sur le lien LNE-SYRTE - LPL, nous avons obtenu d'excellents résultats pour deux longueurs de lien fibré métropolitain : $86 \mathrm{~km}$ et $172 \mathrm{~km}$ (double passage dans la fibre de $86 \mathrm{~km}$ ) [28]. Pour les temps inférieurs à quelques centaines de secondes, la stabilité de fréquence s'améliore proportionnellement au temps d'intégration.

Ces expériences ont permis de valider les liens fibrés sur porteuse optique pour la dissémination de fréquences ultrastables sur des distances de 200 km-300 km. Le développement de liens plus longs a été freiné par la difficulté d'accès à des fibres optiques existantes. Parallèlement à nos travaux, des équipes japonaises ont commencé à travailler dans ce domaine $[29,30]$ et nos collègues du PTB ont eu la possibilité de développer un lien de $920 \mathrm{~km}$ sur fibre dédiée reliant Braunschweig à Munich [31-33]. Ces activités ont permis la réalisation d'un nouveau type d'amplificateur optique [34] et des mesures d'oscillateurs et d'horloges à distance [35-38]. 


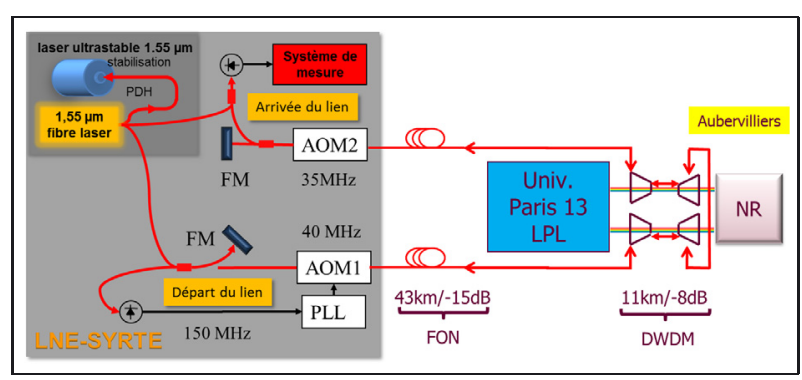

Fig. 5. - Schéma de la liaison optique LNE-SYRTE-LPL Interxion et retour. FON : fibre optique noire, FM : miroir de Faraday, NR : nœud Renater, AOM : Modulateur acousto optique, PDH méthode de stabilisation laser sur cavité optique de Pound Drever Hall.

\section{Transfert de fréquence sur réseau public}

Depuis plus d'une dizaine d'années les opérateurs de télécommunication ont déployé de nombreuses fibres optiques reliant les grandes agglomérations (ossature du réseau ou backbone) de chaque pays et aussi réalisé dans chaque ville un réseau métropolitain avec un maillage très fin. En s'appuyant sur ce réseau, il serait possible en principe de disséminer des signaux métrologiques à grande échelle. Les télécommunications optiques utilisent une gamme spectrale allant de $1528 \mathrm{~nm}$ à $1577 \mathrm{~nm}$, soit $6 \mathrm{THz}$ (bande C). Cette fenêtre est divisée en canaux de $100 \mathrm{GHz}$ chacun $(0,8 \mathrm{~nm})$ pour un fonctionnement en multiplexage en longueur d'onde (DWDM). Un seul de ces canaux est plus que suffisant pour propager le signal métrologique. En 2009, en collaboration étroite avec le Réseau National pour la Technologie, l'Enseignement et la Recherche (RENATER), nous avons effectué un premier essai de partage d'un réseau public de télécommunications avec des signaux optiques métrologiques. Il faut rappeler que la majorité des réseaux de télécommunications sont monodirectionnels, si bien que deux fibres sont utilisées pour les deux sens de propagation. Les signaux optiques qui transportent les données se propagent ainsi dans une seule direction dans chaque fibre.

Or, pour compenser le bruit de phase dû à la propagation nous avons besoin de réaliser un aller-retour « tout optique » sur la même fibre. Il faut donc impérativement contourner tout dispositif optique monodirectionnel $\mathrm{du}$ réseau. De même, pour assurer la continuité optique du signal métrologique, tout équipement électronique doit être également contourné. Afin d'injecter notre signal métrologique dans le réseau fibré de Renater, puis de l'extraire de ce réseau, nous avons utilisé des dispositifs optiques passifs avec trois ports, communément appelés OADM (Optical Add Drop Multiplexer). Ces composants sont capables d'insérer ou d'extraire le signal optique d'un canal spécifique. Grâce à ces composants qui sont bidirectionnels par construction et à faibles pertes d'insertion optiques $(<1 \mathrm{~dB})$ nous pouvons injecter le signal métrologique dans une fibre optique transportant d'autres longueurs d'onde dédiées aux données de l'internet par exemple.

La figure 5 présente le schéma de l'expérience pour laquelle, aux $86 \mathrm{~km}$ de fibres dédiées entre le SYRTE et

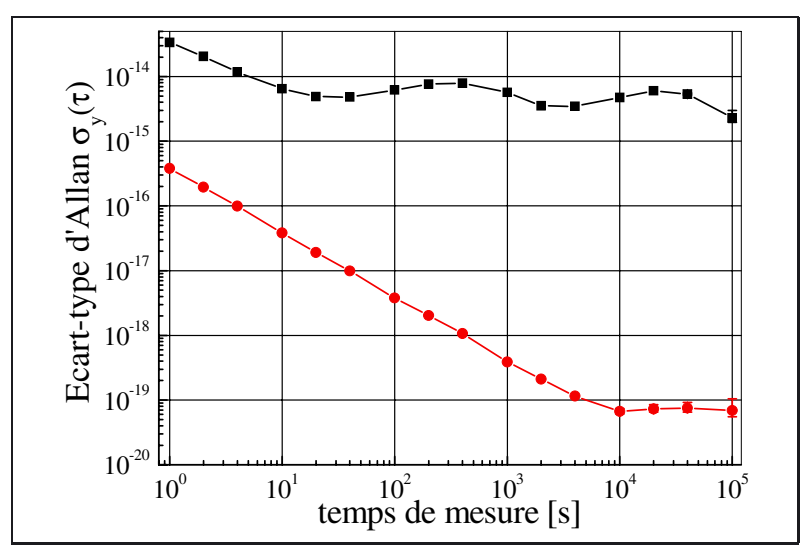

Fig. 6. - Stabilité de fréquence du lien multiplexé de 108 km non stabilisé (carrés noirs) et stabilisé (point rouges).

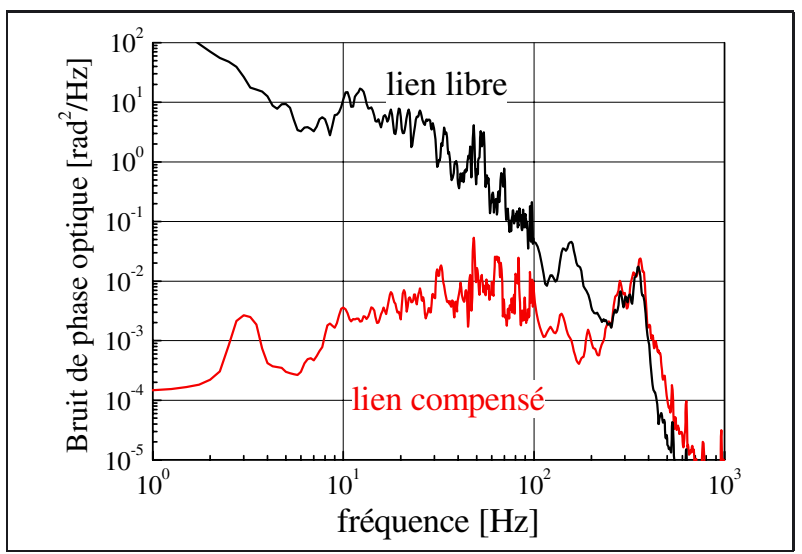

Fig. 7. - Spectre de bruit de phase à $200 \mathrm{THz}(1542 \mathrm{~nm}) \mathrm{du}$ lien optique fibré de $108 \mathrm{~km}$, libre et compensé.

le LPL, nous avons ajouté $2 \times 11 \mathrm{~km}$ de fibres du réseau de télécommunication reliant le Centre de Ressources Informatiques (CRI) de l'Université Paris 13 au Data Center Facility (DCF) Interxion à Aubervilliers. A l'aide de quatre OADM, le signal métrologique à 1542,14 nm (canal ITU 44) est injecté et extrait des fibres qui transportent les données de l'internet sur le canal ITU 34 avec un débit de $10 \mathrm{Gbits} / \mathrm{s}$. Notre premier résultat a été de démontrer que la présence du signal métrologique ne perturbait pas le fonctionnement du réseau internet. La figure 6 présente la stabilité de fréquence du lien multiplexé. La stabilité atteint $3 \times 10^{-16}$ à $1 \mathrm{~s}$ et la stabilité long-terme est d'environ $1 \times 10^{-19}$, limitée par l'interféromètre utilisé pour la mesure de la phase optique [39]. Une légère dégradation est observée par rapport au lien de $86 \mathrm{~km}$, due au bruit additionnel des $22 \mathrm{~km}$ de fibre. La figure 7 présente le spectre en puissance du bruit de phase optique du lien, libre et compensé, sur lequel on observe l'effet du délai qui limite clairement la bande passante à $300 \mathrm{~Hz}$ environ.

Le résultat de cette expérience préliminaire a été décisif et a ouvert la voie à l'utilisation des réseaux publics pour le transfert de signaux métrologiques. Par opposition aux fibres dédiées ou «noires » (dark fiber) des expériences précédentes, ce nouveau concept de transmission 


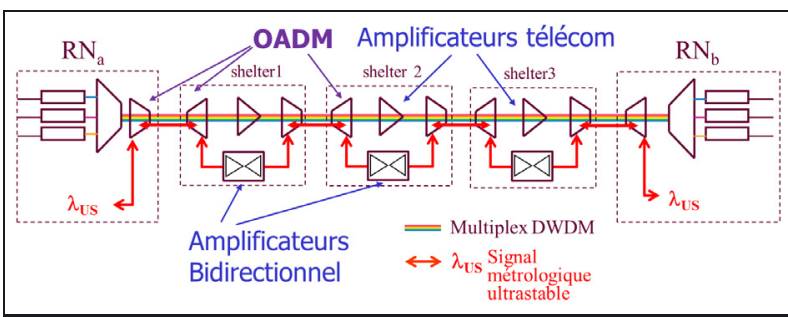

Fig. 8. - Principe de l'architecture d'un lien optique ultrastable sur canal noir ; RN (nœud Renater), $\lambda_{\text {US }}$ (signal optique ultrastable), shelter (abri pour les amplificateurs optiques).

a été appelé «canal noir» (dark channel). L'architecture d'un lien de longue distance basée sur cette technique est présentée sur la figure 8 .

Le signal ultrastable $\lambda_{\text {US }}$ est inséré à l'aide d'un OADM dans le flux de signaux numériques des autres canaux, au niveau d'un nœud du réseau fibré de RENATER. Après propagation dans la première section de fibre et en amont de l'amplification optique par l'équipement du réseau de télécommunications, le signal ultrastable $\lambda_{\text {US }}$ est extrait du flux optique multiplexé au moyen d'un OADM (isolation typique $>20 \mathrm{~dB}$ ), puis amplifié avec un amplificateur bidirectionnel et réinjecté dans la section suivante avec un deuxième OADM.

Cette opération sera répétée tout au long de la fibre. Avec cette approche innovante, nous pouvons tirer avantage des réseaux optiques existants pour réaliser la distribution et la comparaison de signaux ultrastables à l'échelle nationale et potentiellement continentale. Cet avantage a cependant un prix : il nécessite des modifications du réseau public de télécommunications, certes mineures, mais qui introduisent des éléments optiques qui sont en dehors de la responsabilité de l'équipementier réseau et qui produisent des pertes de l'ordre de 1,5 dB par tronçon.

\section{Transfert de fréquence sur réseau public de longue distance}

Pour développer la technique de distribution sur canal noir, RENATER nous a donné la possibilité d'étendre le lien optique à partir du DCF Interxion sur une distance plus conséquente en autorisant un essai sur une liaison opérationnelle nationale reliant Paris à Reims. Dans l'objectif de réaliser des liens sur longue distance, nous avons développé une station optique relais autonome. Cette station permet de relier deux liens indépendants et donc de réaliser une liaison ultrastable de longueur $(2 \times L)$ à partir de deux liens de longueur $L$ en cascade. Cette approche permet de réduire la longueur de chaque lien optique indépendant, ce qui revêt plusieurs avantages : l'atténuation est moindre donc le rapport signal à bruit est amélioré, le délai de propagation est plus court, donc la bande passante de correction est plus élevée ainsi que l'amplitude de réjection du bruit (voir partie 4). La station inclut un laser local de largeur de raie assez fine (inférieure à quelques kilohertz) qui est verrouillé en phase (avec une

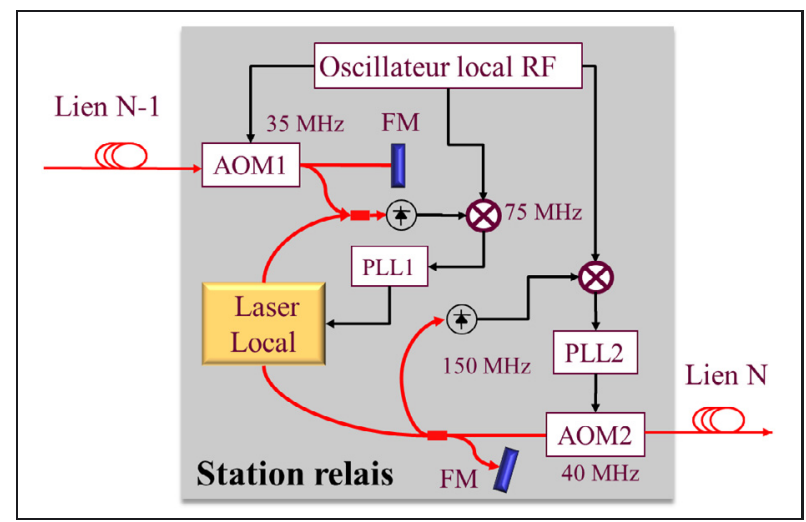

Fig. 9. - Schéma de la station relais, FM : miroir Faraday, AOM : modulateur acousto-optique, PLL : asservissement en phase.

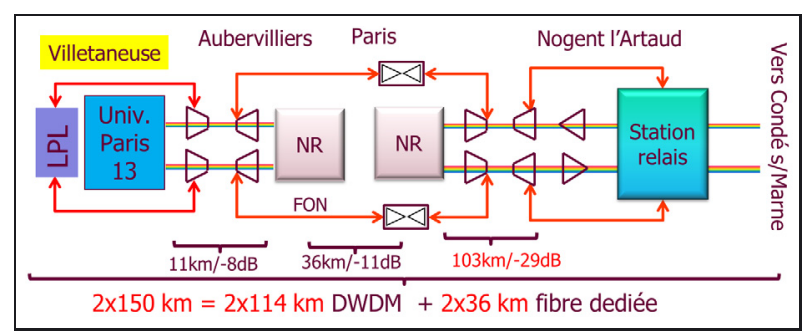

Fig. 10. - Schéma du lien optique Villetaneuse-Nogent l'Artaud-Villetaneuse.

bande passante d'environ $100 \mathrm{kHz}$ ) sur le signal optique entrant. Avec cette approche, nous effectuons une régénération optique du signal ultrastable qui permet de filtrer le bruit large bande ajouté par les amplificateurs optiques. La figure 9 montre le schéma de cette station. On peut y distinguer le laser local (laser à fibre) ainsi que l'AOM utilisé pour corriger son bruit. La station contient également un module optique pour réaliser les traitements des signaux optiques des liens, deux AOM (un pour le lien entrant un pour le lien sortant) et l'électronique RF analogique et numérique pour réaliser le traitement des différents signaux de la station, en particulier le verrouillage automatique du laser local et la correction du bruit du lien sortant.

Cette station a aussi la particularité de ne pas nécessiter d'oscillateur RF local stable, grâce au choix astucieux des fréquences RF des différents asservissements qui permet une compensation exacte du bruit de phase de cet oscillateur local.

Cette station permet de réaliser un lien assez long et dans le même temps peu bruyant et robuste vis-à-vis des diverses perturbations du réseau fibré. Avec le support de RENATER, nous avons testé cette station sur un lien en cascade constitué de deux tronçons de $150 \mathrm{~km}$, entre Villetaneuse et Nogent l'Artaud. La figure 10 présente le schéma de ce lien optique. Une paire de fibres dédiées de $36 \mathrm{~km}$ relient le DCF d'Interxion à un deuxième DCF (Telehouse 2, Paris $11^{\mathrm{e}}$ ), où le signal ultrastable $\lambda_{\text {US }}$ est amplifié dans un amplificateur bidirectionnel qui compense les pertes optiques. À l'aide d'un OADM, le signal 


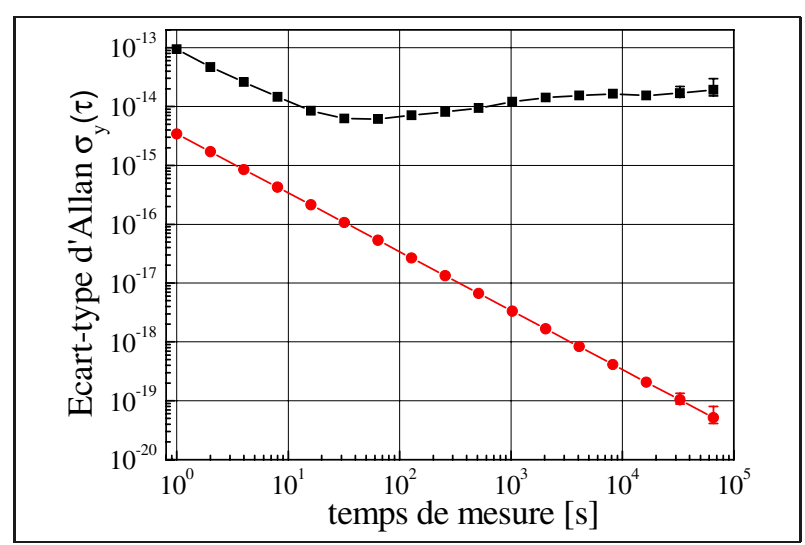

Fig. 11. - Stabilité de fréquence du lien de $(2 \times 150) \mathrm{km}$ non compensé (carrés noirs), et activement stabilisé (points rouges).

optique métrologique ultrastable est ensuite inséré sur la liaison optique opérationnelle de Paris à Nancy via Reims. Cette liaison opérationnelle occupe deux canaux (42 et 43, centrés sur les longueurs d'onde $1542,9 \mathrm{~nm}$ et $1543,7 \mathrm{~nm}$ ) adjacents à celui du signal métrologique (44). Le premier amplificateur optique de cette liaison de télécommunication est situé dans un «abri » (shelter) à Nogent l'Artaud après 103 km de fibre.

À Nogent l'Artaud, le signal ultrastable est extrait à l'aide d'un OADM et traité par la station relais automatique. Le signal de sortie de cette station est envoyé vers Paris sur la deuxième fibre du réseau RENATER. À Paris le signal est extrait, amplifié et envoyé vers Interxion puis vers le LPL à Villetaneuse où la stabilité de fréquence du signal optique ayant parcouru les $300 \mathrm{~km}$ est mesurée. La figure 11 présente la stabilité de ce lien. Le bruit du lien libre est considérablement augmenté par rapport à la liaison plus courte de $108 \mathrm{~km}$. Néanmoins, après correction du bruit de propagation, la stabilité en boucle fermée atteint quelques $10^{-15}$ à $1 \mathrm{~s}$ (pour une bande passante de mesure de $10 \mathrm{~Hz}$ ) pour franchir la gamme des $10^{-19}$ audelà de $30000 \mathrm{~s}$ [40]. Au-delà de cette performance, il faut souligner que le fonctionnement du réseau de télécommunication n'a pas été affecté par le signal métrologique et c'est ainsi la première fois qu'un signal métrologique a pu être propagé sur une liaison opérationnelle intercités.

Ce résultat a démontré la fiabilité de la technique de lien optique sur canal noir et ouvert la voie au déploiement de liens de longues distances. Cependant, il a fallu d'abord résoudre plusieurs points durs susceptibles de limiter la distance couverte et la robustesse du lien. Par exemple, en présence de segments avec des pertes importantes, le faible signal optique d'aller-retour peut être noyé dans le bruit d'émission spontanée amplifiée des amplificateurs à fibre dopée à l'erbium. De plus, les fluctuations de l'état de polarisation dans une fibre monomode peuvent provoquer une extinction du battement avec le laser local.

Nous avons donc réalisé un important travail d'amélioration du système pour le rendre opérationnel sur des distances encore plus importantes et sur des périodes

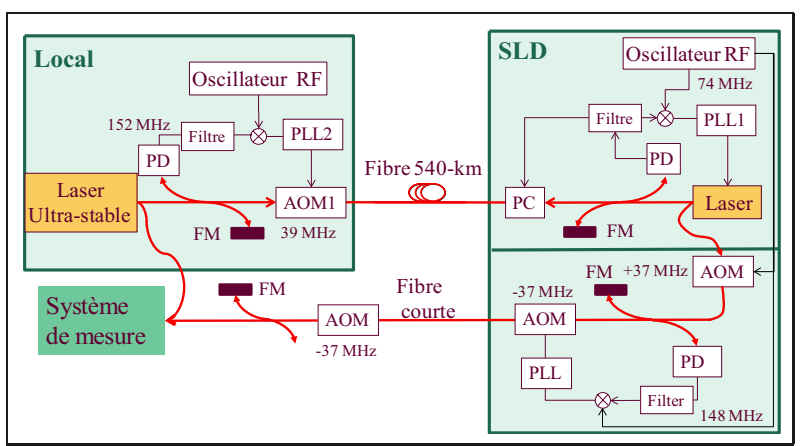

Fig. 12. - Synoptique du lien optique de $540 \mathrm{~km}$ avec schéma fonctionnel de la Station Laser Distante (SLD).

beaucoup plus longues. Nous avons pour cela développé une nouvelle génération de station de régénération dans lesquelles le signal optique du laser local est non seulement utilisé pour injecter le lien sortant en aval mais est aussi renvoyé vers la station en amont. Ceci est équivalent à une très forte amplification, par exemple le gain est de $60 \mathrm{~dB}$ dans le cas où la puissance optique reçue est de l'ordre du nanowatt et la puissance réinjectée dans la fibre de l'ordre du milliwatt. Nous avons également implémenté un contrôle automatique de la polarisation pour maximiser la puissance du battement avec le laser local.

Le synoptique des équipements de traitement du signal ultrastable est présenté sur la figure 12. On peut y distinguer le départ du lien (local) et la station laser distante (SLD sur fig. 12), tous les deux localisés au LPL. Le laser local est une diode laser à bas bruit (largeur de raie $<10 \mathrm{kHz}$ ), verrouillée en phase sur le signal entrant par rétroaction sur le courant avec un bande passante de $100 \mathrm{kHz}$. La station SLD inclut un système de contrôle automatique de l'état de polarisation entrant, qui, en fonction d'un seuil prédéfini, est optimisé pour maximiser le battement avec l'oscillateur optique local.

La réalisation technique de cette station a été également optimisée : ainsi le module optique est maintenant réalisé sur mesure avec des fibres soudées et inséré dans un boitier thermalisé (fig. 13).

Avec cette station nous avons pu démontrer un lien de $540 \mathrm{~km}$ sans station relais intermédiaire entre Villetaneuse, Reims et Villetaneuse. Cette liaison de $540 \mathrm{~km}$ a été obtenue en ajoutant deux nouvelles sections de réseau fibré au lien précédent de $300 \mathrm{~km}$.

La figure 14 présente la structure de ce lien optique, pour lequel l'atténuation optique dépasse $160 \mathrm{~dB}$. Seize modules OADM sont utilisés pour extraire et réinsérer le signal ultrastable tout au long du lien et six amplificateurs bidirectionnels sont utilisés pour compenser partiellement les pertes et les réduire à $60 \mathrm{~dB}$. Le gain des amplificateurs bidirectionnels est cependant limité autour de $13 \mathrm{~dB}$ à $20 \mathrm{~dB}$ par les réflexions parasites et la diffusion Rayleigh, si bien que les pertes résiduelles restent assez élevées.

Avec ce lien, qui ne perturbe pas le fonctionnement du réseau Internet RENATER, nous avons démontré une 


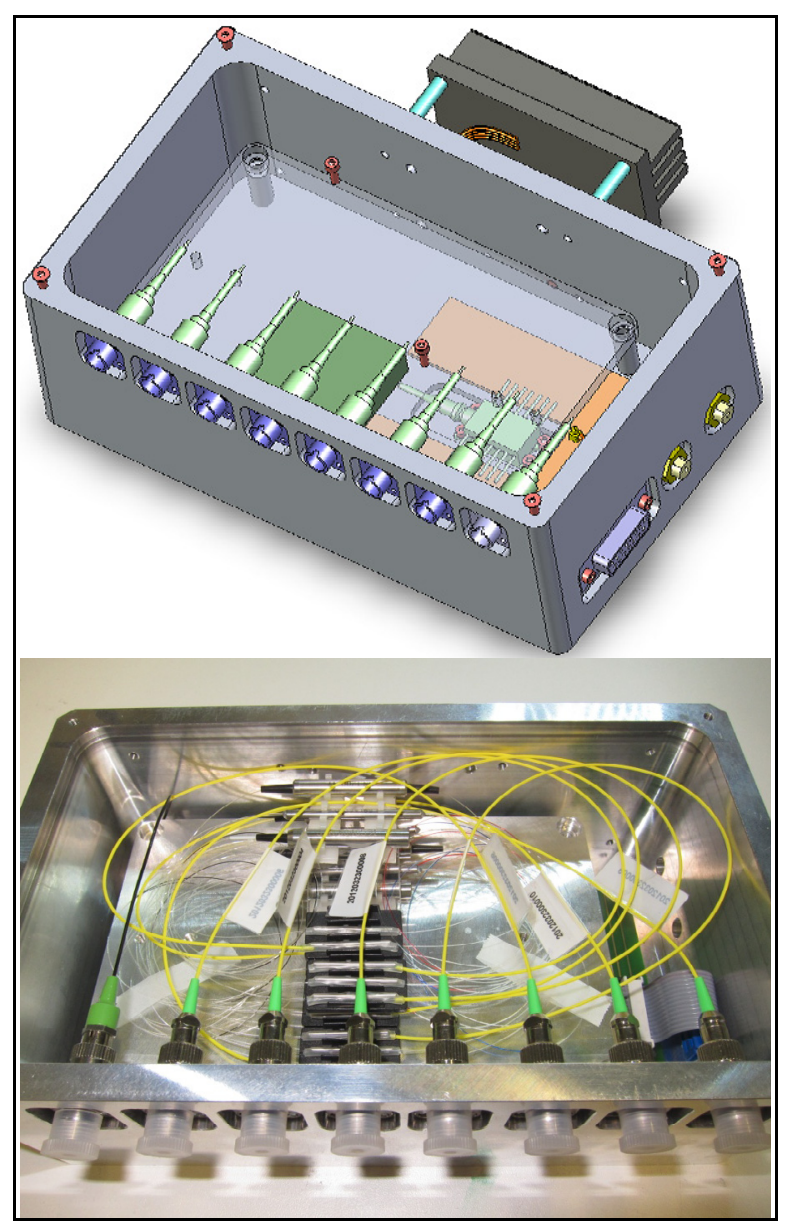

Fig. 13. - Schéma et photographie du module optique de la station.

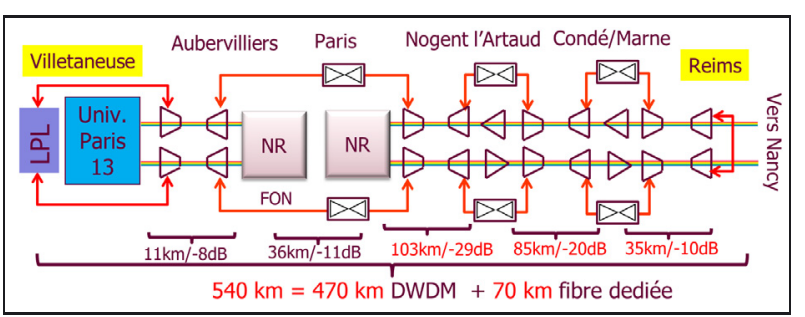

Fig. 14. - Schéma du lien optique de 540 km VilletaneuseReims-Villetaneuse avec les atténuations optiques pour chaque segment; RN : nœud Renater.

stabilité de fréquence de $5 \times 10^{-15}$ pour un temps de mesure de $1 \mathrm{~s}$ (dans une bande passante de mesure de $10 \mathrm{~Hz}$ ) et meilleure que $10^{-18}$ au-delà de $10000 \mathrm{~s}$ (fig. 15).

Nous avons aussi vérifié que ce lien optique n'introduit pas de biais de fréquence. La valeur moyenne de la fréquence détectée est compatible avec la valeur attendue compte tenu de l'incertitude déterminée par l'écart type d'Allan [22]. La résolution de ce transfert de fréquence est meilleure de plus d'un ordre de grandeur que le niveau de reproductibilité des meilleures horloges optiques actuelles. Elle excède de trois ordres de grandeur les meilleurs étalons primaires et enfin représente un gain de 4 à 5 ordres de grandeur par rapport au système GPS.

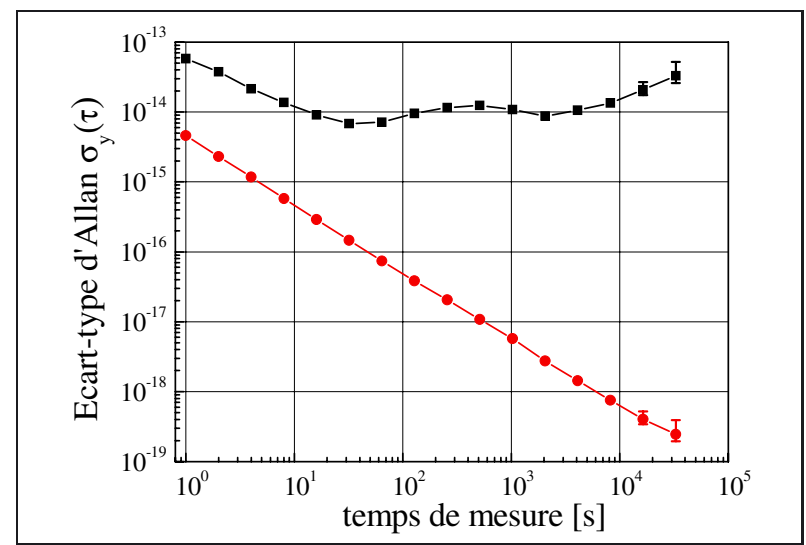

Fig. 15. - Stabilité du lien de $540 \mathrm{~km}$ non compensé (carrés noirs) et stabilisé (points rouges).

Tableau 1

Bilan des résultats des liens optiques fibrés.

\begin{tabular}{|c|c|c|}
\hline Lien optique fibré & $\begin{array}{l}\text { Stabilité } \\
\text { (1 s) }\end{array}$ & $\begin{array}{c}\text { Stabilité } \\
\left(10^{4} \mathrm{~s}\right)\end{array}$ \\
\hline $\begin{array}{l}\text { Transfert micro-onde } \\
1 \mathrm{GHz} \text { sur } 86 \mathrm{~km}\end{array}$ & $5 \times 10^{-15}$ & $1 \times 10^{-17}$ \\
\hline $\begin{array}{l}\text { Transfert micro-onde } \\
9 \mathrm{GHz} \text { sur } 86 \mathrm{~km}\end{array}$ & $1,2 \times 10^{-15}$ & $1 \times 10^{-18}$ \\
\hline $\begin{array}{l}\text { Transfert optique } \\
\text { sur } 86 \mathrm{~km}\end{array}$ & $3 \times 10^{-16}$ & $2 \times 10^{-19}$ \\
\hline $\begin{array}{c}\text { Transfert optique } \\
172 \mathrm{~km}\end{array}$ & $3 \times 10^{-16}$ & $2 \times 10^{-19}$ \\
\hline $\begin{array}{c}\text { Transfert optique } \\
\text { lien multiplexé de } 108 \mathrm{~km}\end{array}$ & $4 \times 10^{-16}$ & $<10^{-19}$ \\
\hline $\begin{array}{c}\text { Transfert optique } \\
\text { lien multiplexé de }(2 \times 150) \mathrm{km}\end{array}$ & $3 \times 10^{-15}$ & $3 \times 10^{-19}$ \\
\hline $\begin{array}{c}\text { Transfert optique } \\
\text { lien multiplexé de } 540 \mathrm{~km}\end{array}$ & $6 \times 10^{-15}$ & $8 \times 10^{-19}$ \\
\hline
\end{tabular}

Ces derniers résultats représentent un pas significatif vers un lien sur longue distance qui utilise un canal optique DWDM d'un réseau public de télécommunications. Ils valident les briques élémentaires d'un réseau pour la dissémination et la comparaison de références optiques ultrastables sur une échelle continentale.

\section{Conclusion et perspectives}

Nous avons présenté les résultats d'une décennie d'études sur le transfert de fréquence sur fibre optique. Le tableau 1 résume ces résultats.

Les résultats obtenus avec le transfert par modulation d'amplitude sur une distance approchant la centaine de kilomètres ont ouvert la voie au transfert par fibre optique de références de fréquence de qualité métrologique. La démonstration du tout premier lien optique sur longue distance exploitant la phase optique sur des distances significatives (autour de $100 \mathrm{~km}$ ) a été suivie d'une multitude d'expériences dans plusieurs pays d'Europe, aux Etats-Unis, au Japon, en Australie, en Chine... Le domaine est maintenant mûr et le défi actuel est de transformer ces expériences en outils capables de contribuer 


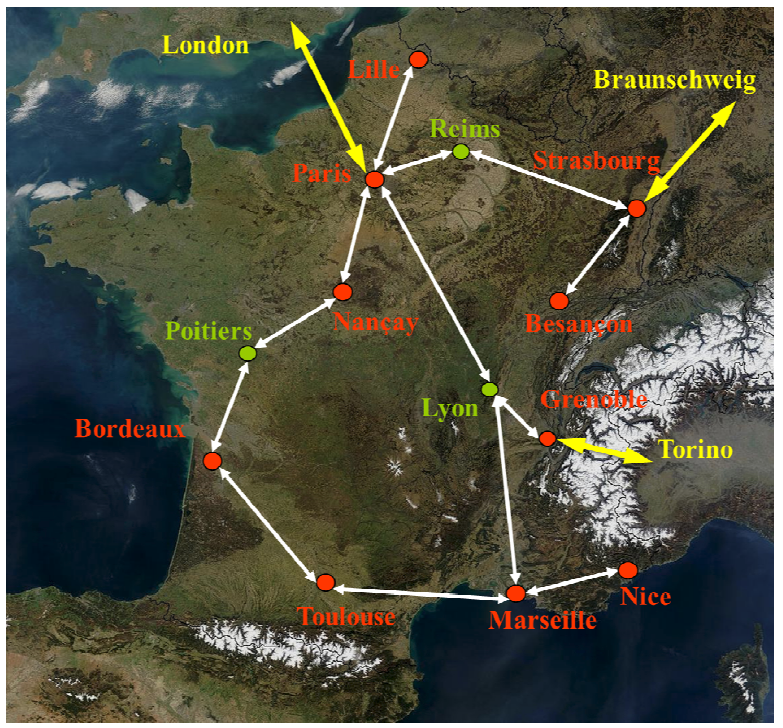

Fig. 16. - Schéma du réseau optique du projet REFIMEVE+.

de façon opérationnelle au développement de la métrologie des fréquences au plus haut niveau. Ceci requiert des liaisons optiques pérennes et fiables au niveau national et international entre les instituts nationaux de métrologie. Au niveau européen, un projet coordonné de métrologie JRP (Joint Research Program) NEAT-FT est en train d'organiser la recherche autour de ce sujet. Au niveau national, le développement d'un réseau français ultrastable a été financé récemment dans le cadre des investissements d'avenir (Equipements d'Excellence EquipEx). Ce projet appelé REFIMEVE+ (REseau FIbré MEtrologique à Vocation Européenne) a pour objectif la construction d'un réseau fibré utilisant le support de l'internet géré par RENATER et permettant le transfert d'une référence de fréquence optique ultrastable sans dégradation des performances sur des distances de plusieurs milliers de kilomètres. Ce réseau connectera 19 partenaires scientifiques, 8 en région parisienne et 11 situés dans différentes villes françaises. Il sera réalisé en partenariat avec une PME. La figure 16 montre un schéma simplifié du déploiement du réseau REFIMEVE+.

Les laboratoires utilisateurs pourront dès lors disposer d'une référence absolue de fréquence ayant la même exactitude que la référence de fréquence délivrée par le LNE-SYRTE. De multiples usages s'offriront à eux. Grâce à l'utilisation d'un peigne de fréquences ils pourront ramener la fréquence ultrastable de $194,4 \mathrm{THz}$ (1542 nm) fournie à la fréquence de leur choix, optique, radiofréquence ou microonde. Ils pourront ainsi contrôler les fréquences lasers intervenant dans de nombreuses expériences de physique atomique ou moléculaire à des fins de spectroscopie ou de mesures de très haute précision. À partir du réseau existant, il sera toujours possible de connecter d'autres laboratoires académiques ou industriels si une liaison par fibre optique est disponible. Le réseau lui-même pourra être un objet d'étude. En effet, comme le montre la figure 16, il forme une boucle géante dont la surface excède les $100000 \mathrm{~km}^{2}$. Les signaux lasers ultrastables circulant dans des sens opposés dans cette boucle présenteront une différence de phase due à l'effet Sagnac, proportionnelle à cette surface géante. L'effet Sagnac étant dû à la rotation de la Terre. Cette différence de phase pourra être étudiée pour mettre en évidence différents effets géophysiques [41].

Ce projet très ambitieux présente un volet international dont les enjeux sont majeurs pour la métrologie européenne et mondiale. Début 2015 devrait être réalisée une première connexion entre le LNE-SYRTE et le PTB à Braunschweig. Ce projet permettra de réaliser pour la première fois une comparaison des meilleurs étalons de fréquence français et allemands grâce à un transfert de fréquence sur plus de $1500 \mathrm{~km}$. Ceci ouvrira la voie aux comparaisons, en temps réel et en continu, de grandes variétés d'étalons de fréquence développés dans plusieurs pays sans que la comparaison soit limitée par le transfert de fréquence.

Ces développements futurs sont de nature à influencer profondément la façon de faire de la communauté internationale en métrologie des fréquences. Un autre champ d'applications concerne la comparaison des horloges terrestres et spatiales notamment au travers des projets PHARAO-ACES dont les horloges devraient être envoyées en 2016 à destination de la station spatiale internationale et grâce auxquelles des tests de la relativité générale pourront être réalisés à des précisions inégalées.

Enfin, bien que les résultats soient encore préliminaires, il est aussi probable que le transfert d'une échelle de temps de référence par fibre optique sera dans les années à venir source de développements majeurs de la métrologie du temps [42].

\section{Remerciements}

Les auteurs tiennent à remercier les différents doctorants et post doctorants qui ont contribué très largement à tous ces développements. Ces travaux ont bénéficié du soutien du LNE, du CNRS, de l'Université Paris 13, de l'Agence Nationale de la Recherche et de la région Îlede-France. Les auteurs remercient également le Centre de ressources Informatiques de l'Université Paris 13 pour leur avoir permis d'accéder au réseau Renater. Enfin, ce projet n'aurait pas pu connaître ces développements récents sans le précieux concours de Renater et la grande disponibilité de ses ingénieurs.

\section{Références}

[1] Li R., GibBle K. et Szymaniec K., "Improved accuracy of the NPL-CsF2 primary frequency standard: evaluation of distributed cavity phase and microwave lensing frequency shifts," Metrologia, 48, 2011, 283-289.

[2] Guena J., Abgrall M., Rovera D., Laurent Ph., Chupin B., Lours M., SANTARElli G., Rosenbusch P., Tobar M.E., Li R., Gibble K., Clairon A., Bize S., Cacciapuoti L., Chambon D., Luiten A., Tobar M. et SALOMON C., "Progress in atomic fountains at LNESYRTE", I.E.E.E. Trans. Ultra., Ferro., Frequ. Control, 59, 2012, 394-410.

[3] Weyers S., Gerginov V., Nemitz N., Li R. et GibBle K., "Distributed cavity phase frequency shifts of the caesium fountain PTB-CSF2", Metrologia, 49, 2012, 82-87. 
[4] Swallows M.D., Bishof M., LiN Y., BlatT S., MaRTIN M.J., REY A.M. et YE J., "Suppression of Collisional Shifts in a Strongly Interacting Lattice Clock", Science, 331, 6020, 2011, 1043-1046.

[5] Katori H., "Optical lattice clocks and quantum metrology”, Nature Photonic, 5, 2011, 203-210.

[6] HuntemanN N., OKhapkin M., LipPhaRdT B., WeYers S., TAMm Chr. et PEIK E., "High-Accuracy Optical Clock Based on the Octupole Transition in ${ }^{171} \mathrm{Yb}^{+}$, Phys. Rev. Lett., 108, 2012, 153002-5.

[7] Sherman J.A., LEMKe N.D., Hinkley N., Pizzocaro M., Fox R.W., Ludlow A.D. et OATES C.W., "HighAccuracy Measurement of Atomic Polarizability in an Optical Lattice Clock", Phys. Rev. Lett., 108, 2012, 153002-5.

[8] Ashby N., Heavner T.P., JefFerts S.R, Parker T., RADNAEV A.G. et DUDIN Y.O., "Testing local position invariance with four cesium-fountain primary frequency standards and four NIST hydrogen masers", Phys. Rev. Lett., 98, 2007, 070802.

[9] Shelkovnikov A., Butcher R.J., Chardonnet Ch. et AMY-KLEIN A., "Stability of the Proton-to-Electron Mass Ratio" Phys. Rev. Lett., 100, 2008, 150801.

[10] Chou C., Hume D.B., Rosenband T. et Wineland D.J., "Optical Clocks and Relativity", Science, 329, 2010, 1630-1633.

[11] Guena J., Abgrall M., Rovera D., Tobar M.E., Laurent Ph., Clairon A. et Bize S., "Improved Tests of Local Position Invariance Using ${ }^{87} \mathrm{Rb}$ and ${ }^{133} \mathrm{Cs}$ Fountains", Phys. Rev. Lett., 109, 2012, 080801.

[12] Bauch A., AchKar J., Bize S., Calonico D., Dach R., Hlavać R., Lorini L., PARker T., Petit G., Piester D., SZYMANIEC K. et Uhrich P., "Comparison between frequency standards in Europe and the USA at the $10^{-15}$ uncertainty level", Metrologia, 43, 2006, 109-120.

[13] Calhoun M., Huang S. et TJoelker R.L., "Stable Photonic Links for Frequency and Time Transfer in the Deep-Space Network and Antenna Arrays", Proc. of the I.E.E.E., Special Issue on Technical Advances in Deep Space Communications $\mathcal{E}$ Tracking, 95, 2007, 1931-1946.

[14] Foreman S.M., Holman K.W., Hudson D.D., JoNes D.J. et YE J., "Remote transfer of ultrastable frequency references via fiber networks", Rev. Sci. Instrum., 78, 2007, 021101.

[15] Daussy Ch., Lopez O., Amy-Klein A., Goncharov A., Guinet M., Chardonnet Ch., Narbonneau F. Lours M., Chambon D., Bize S., Clairon A. et SANTARELli G., TOBAR M.E. et LUITEN A.N., "Longdistance frequency dissemination with a resolution of 10-17", Phys. Rev. Lett., 94, 2005, 203904.

[16] Narbonneau F., Lours M., Bize S., Clairon A., SANTARElli G., LOPEZ O., DAUSSY Ch., AMY-KleIN A et CHARDONNET Ch., "High resolution frequency standard dissemination via optical fiber metropolitan network", Rev. Sci. Instrum., 77, 2006, 064701.

[17] Lopez O., Amy-Klein A., Daussy Ch., Chardonnet Ch., Narbonneau F., Lours M. et SANTARElli G., "86-km optical link with a resolution of $2 \times 10^{-18}$ for RF frequency transfer", Eur. Phys. J. D, 48, 2008, 35-41.
[18] Lopez O., Lours M., Amy-Klein A., Chardonnet Ch. et SANTARELli G., "High-resolution microwave frequency dissemination on an 86-km urban optical link", Appl. Phys. B: Lasers Opt., 98, 2010, 723-727.

[19] Kumagai M., Fujieda M., Nagano S. et Hosokawa M., "Stable radio frequency transfer in $114 \mathrm{~km}$ urban optical fiber link", Opt. Lett., 34, 2009, 2949-2951.

[20] Fujieda M., Kumagai M., Gotoh T. et Hosokawa M., "Ultrastable Frequency Dissemination via Optical Fiber at NICT", I.E.E.E. Trans. Instrum. Measur, 58, 2009, 1223-1228 et FuJieda M., Kumagai M., et NAGANo S., "Coherent Microwave Transfer Over a 204-km Telecom Fiber Link by a Cascaded System", I.E.E.E. Transactions on Ultra., Ferro., Frequ. Control, 57, 2010, 168-174.

[21] Grosche G. et al., "Transmission of an Optical Carrier Frequency over a Telecommunication Fiber Link", Conference on Lasers and Electro-Optics/Quantum Electronics and Laser Science Conference and Photonic Applications Systems Technologies, OSA Technical Digest (CD) (Optical Society of America, 2008, CMKK1.

[22] Lopez O., Haboucha A., Chanteau B., Chardonnet Ch., AMY-KLEIN A. et SANTARELli G., "Ultra-stable long distance optical frequency distribution using the Internet fiber network", Opt. Express, 20, 2012, 23518-23526.

[23] PREDEHL K. et al., "A 920-Kilometer optical fiber link for frequency metrology at the 19th decimal place", Science, 336, 2012, 441-444.

[24] ŚLIWCZYŃsKi Ł., KREHLIK P., BuczeK Ł. et LiPIŃSKI M., "Frequency transfer in electronically stabilized fiber optic link exploiting bidirectional optical amplifiers", I.E.E.E. Trans. Instrum. Measur., 1-8, DOI: 10.1109/ TIM. 2012.2188663N (2012).

[25] Ma L.-S., Jungner P., Ye J. et HALl J.L., "Delivering the same optical frequency at two places: accurate cancellation of phase noise introduced by an optical fiber or other time-varying path", Opt. Lett., 19, 1994, 1777-1779.

[26] De Beauvoir B., NeZ F., Hilico L., JULIEN L., BiRABEN F., Cagnac B., Zondy J.-J., Touahri D., ACEF O. et Clairon A., "Transmission of an optical frequency through a $3 \mathrm{~km}$ long optical fiber", Eur. Phys. J. D, 2, 1998, 227-229.

[27] Newbury N.R., Williams P.A. et SWANN W.C., "Coherent transfer of an optical carrier over $251 \mathrm{~km}$ ", Opt. Lett., 32, 2007, 3056 et Williams P.A., SWANN W.C, NEWBURY N.R., "High-stability transfer of an optical frequency over long fiber-optic links", J. Opt. Soc. Am. B, 25, 2008,1284 .

[28] JIANG H. et al., "Long-distance frequency transfer over an urban fiber link using optical phase stabilization", J. Opt. Soc. Am. B, 25, 2008, 2029-2035.

[29] Musha M., Hong F., Nakagawa K. et Ueda K., "Coherent optical frequency transfer over $50-\mathrm{km}$ physical distance using a 120-km-long installed telecom fiber network", Opt. Express, 16, 2008, 16459.

[30] Fujieda M., Kumagai M., Nagano S. Yamaguchi A., HACHISU H. et IDO T., "All-optical link for direct comparison of distant optical clocks", Opt. Express, 19, 2010, 16498. 
[31] Grosche G. et al., "Optical frequency transfer via $146 \mathrm{~km}$ fiber link with $10^{-19}$ relative accuracy", Opt. Lett., 34, 2009, 2270-2272.

[32] TERRA O. et al., "Phase-coherent comparison of two optical frequency standards over $146 \mathrm{~km}$ using a telecommunication fiber link", Appl. Phys. B, 97, 2009, 541-551.

[33] Schnatz H. et al., "Phase-Coherent Frequency Comparison of Optical Clocks Using a Telecommunication Fiber Link", IEEE Transactions on Ultrasonics, Ferroelectrics, and Frequency Control, 57, 2010, 175-181.

[34] Terra O., Grosche G. et Schnatz H., "Brillouin amplification in phase coherent transfer of optical frequencies over 480 km fiber", Opt. Express, 18, 2010, 16102-16111.

[35] HoNG F. et al., "Measuring the frequency of a Sr optical lattice clock using a $120 \mathrm{~km}$ coherent optical transfer," Opt. Lett., 34, 2009, 692-694.

[36] PAPE A. et al., "Long-distance remote comparison of ultrastable optical frequencies with $10^{-15}$ instability in fractions of a second", Opt. Express, 18, 2010, 21477-21483.

Article reçu le 21 février 2013, version révisée reçue le 11 février 2014.
[37] Yamaguchi A. et al., "Comparison of distant optical lattice clocks at the $10^{-16}$ uncertainty", Appl. Phys. Express, 4, 2011, 082203.

[38] FRIEBE, J. et al., "Remote frequency measurement of the ${ }^{1} \mathrm{~S}_{0} \rightarrow{ }^{3} \mathrm{P}_{1}$ transition in laser-cooled ${ }^{24} \mathrm{Mg}$ ", New J. Phys., 13, 2011, 125010 .

[39] Kéfélian F., Lopez O., Jiang H., Chardonnet Ch., AMY-KLEIN A. et SANTARELli G., "High-resolution optical frequency dissemination on a telecommunication network with data traffic", Opt. Lett., 34, 2009, 1573-1575.

[40] Lopez O., Haboucha A., Kéfélian F., Jiang H., Chanteau B., Roncin V., Chardonnet Ch, AmYKLein A. et SANTARELli G., "Cascaded multiplexed optical link on a telecommunication network for frequency dissemination", Opt. Express, 18, 2010, 16849-16857.

[41] Clivati C., Calonico D., Costanzo G.A., Mura A., Pizzocaro M., LeVI F., "A Large Area Fiber Optic Gyroscope on multiplexed fiber network", arXiv: 12125717, 2012.

[42] Lopez O., Kanj A., Pottie P., Rovera D., AchKar J., Chardonnet Ch., AmY-Klein A. et Santarelli G., "Simultaneous remote transfer of accurate timing and optical frequency over a public fiber network", Appl. Phys. B: Lasers Opt., 110, 2013, 3-6. 